\title{
¿Cuál es el alcance de una teoría de la justicia?
}

\author{
Gustavo PEREira \\ Facultad de Humanidades y Ciencias de la Educación \\ Universidad de la República (Uruguay) \\ hmodzele@adinet.com.uy
}

\begin{abstract}
Resumen: A partir de las críticas que Sen ha realizado a las teorías de la justicia, es posible proyectar tres facetas del alcance de tales teorías. La primera faceta consiste en conjugar un momento idealizado con uno de aplicabilidad, ya que contar con criterios normativos idealizados posibilita orientar la aplicabilidad del modelo. La segunda la constituyen las exigencias de la aplicabilidad, y en esta faceta la base de información de las capacidades que aporta Sen permite hacer una expansión de la justicia rawlsiana. La tercera muestra la necesidad de articular dos bases de información con sus respectivas lógicas distributivas y compensatorias. Estas tres facetas del alcance de una teoría de justicia permiten realizar la justicia en las sociedades democráticas, especialmente en aquellas afectadas por la pobreza, la marginación y las desigualdades sociales.
\end{abstract}

Palabras clave: justicia, igualdad, capacidades

\begin{abstract}
Sen's criticisms to the theories of justice give way to the projection of three aspects of the scope of such theories. The first aspect is the possibility for an idealized moment and one of applicability to work together, because idealized principles and normative criteria allow for the guidance towards the applicability of the model. The second aspect is connected to the demands of applicability, in which task Sen's capability informational basis allows for the expansion of Rawlsian justice. The third aspect presents the articulation of two informational bases and their respective distributive and compensatory logics. These three aspects of the scope of a theory of justice represent the best way toward the realization of justice in democratic societies, especially those severely affected by poverty, marginalization and social inequalities.

Key words: justice, equality, capabilities
\end{abstract}

Existe un consenso relativamente amplio en torno a que las sociedades democráticas tienen como rasgo distintivo el contar con un conjunto de instituciones que se encargan de distribuir las cargas y los beneficios de la cooperación social. La enorme influencia que ha tenido la obra de John Rawls desde la publicación de Teoría de la justicia es responsable en gran medida de dicho consenso. Recordemos que, para Rawls, la sociedad se entiende como una empresa cooperativa que permite obtener ventajas mutuas para sus miembros, y que en ella conviven tanto una identidad de intereses como el conflicto. La convergencia de intereses 
se debe a que, como consecuencia de la cooperación social, las personas pueden tener una vida mejor que la que tendrían sin ella, mientras que el conflicto de intereses se manifiesta en que, con el objetivo de poder alcanzar sus fines, las personas prefieren tener una participación mayor que una menor en los beneficios producidos (cfr. Rawls 1979, p. 21). Son necesarios, entonces, los principios de justicia social para decidir la forma de distribuir los beneficios y las cargas de la cooperación social.

Si bien, como decía al inicio, el acuerdo en estos puntos es amplio, puede afirmarse que se diluye una vez que nos planteamos el alcance que una teoría de justicia debería tener, y es Amartya Sen quien se opone decididamente a la perspectiva de recurrir a principios distributivos. La razón más importante para ello, aduce, es que un programa basado en principios que permitan diseñar instituciones que provean arreglos sociales justos compromete la identificación de estadios intermedios a través de los cuales se pueda ir acercando al ideal de justicia invocado.

En este trabajo, mi objetivo principal es establecer el alcance de una teoría de la justicia, y lo haré presentando tres facetas suyas: a) la relación entre un modelo ideal de justicia y su aplicabilidad; b) la ampliación de la justicia como equidad — planteada por Rawls - a través de un mayor compromiso con su realizabilidad, y c) la articulación de dos bases de información en una misma propuesta. Para ello, examinaré la posición que Amartya Sen defiende en The Idea of Justice (2009) y sostendré que su presentación dicotómica de las perspectivas que identifican un ideal de justicia y de las que realizan una ordenación de arreglos sociales no es lo suficientemente precisa para explicar algunas de las propuestas más influyentes de la discusión sobre justicia distributiva, como son los casos de la justicia como equidad de Rawls y de la igualdad de recursos de Dworkin. A diferencia de Sen, propondré que el conjugar un ideal de justicia con instancias de aplicabilidad sensibles a las circunstancias establece una primera faceta del alcance de una teoría de la justicia que, además de ser teóricamente posible, es el camino más sustentable para asegurar su aplicabilidad.

Por otra parte, sostendré que la base de información de las capacidades que aporta Sen tiene la ventaja de ser altamente sensible a situaciones que no han sido consideradas adecuadamente por la mayoría de las posiciones que han intervenido en el debate sobre la justicia distributiva, y esto tiene importantes consecuencias en la aplicación de programas de justicia social en los países más pobres. Las situaciones de pobreza extrema y marginación que afectan a un porcentaje enorme de la población mundial nos demandan ampliar el alcance de la justicia para que se las pueda combatir exitosamente. La justicia rawlsiana, 
al referirse a las sociedades democráticas como aquellas a las que es preciso conducir a lo que estipula su ideal de justicia, no considera con suficiente precisión algunas características distintivas de la mayoría de dichas sociedades, como la pobreza o la deficiencia de capacidades, que son obstáculos para poder ser un ciudadano pleno, lo que disminuye las posibilidades de realizar el ideal de justicia. A partir de esto postularé que la segunda faceta del alcance de una teoría de la justicia consiste en la necesidad de que sea efectivamente aplicable en sociedades democráticas con los rasgos indicados. El enfoque de las capacidades y su alta sensibilidad a cómo las circunstancias influyen en los resultados de las personas es un candidato apropiado para esta tarea; en tal sentido, introduciré un principio de justicia que pautará la expansión de la justicia rawlsiana tomando la base de información de las capacidades.

Por último, propondré que la expansión de la justicia rawlsiana permite sustentar una propuesta de justicia distributiva de doble base de información, ya que en los casos en que las personas afectadas se encuentren por debajo del umbral de ser plenamente cooperantes operará según una lógica propia de la base de información de las capacidades, y en los casos en que las personas superen tal umbral, con una lógica propia de la base de información de medios —en el caso de Rawls, los bienes primarios-. Esto le da mayor precisión y sensibilidad a las posibilidades de aplicación, y constituye la tercera faceta del alcance de una teoría de la justicia.

A continuación presentaré, como primera parte de la exposición, la postura de Sen sobre las teorías de la justicia.

\section{Ideal de justicia y aplicabilidad}

En su reciente libro The Idea of Justice, Sen discute el alcance de las teorías de la justicia (cfr. Sen 2009) y distingue dos formas de enfrentar y explicar los problemas de la justicia social, a las que denomina el enfoque trascendental y el comparativo. El enfoque trascendental, dice Sen, se focaliza en identificar con perfección los arreglos sociales que constituirán una sociedad justa; el enfoque comparativo, por su parte, se concentra en ordenar alternativas de arreglos sociales, más que en la identificación de una sociedad justa.

La tesis de Sen es que entre ambos enfoques hay una brecha tal que los vuelve irreconciliables, por lo que optar por uno de ellos implica excluir al otro. La razón principal que esgrime es que el enfoque trascendental, para cumplir con sus objetivos, requiere que se aseguren todos los requisitos de una sociedad completamente justa; en conse- 
cuencia, quedan excluidos los avances o etapas intermedias que pueden irse alcanzando en nombre de la justicia, y que se pueden identificar mediante la comparación de posibles alternativas y la elección de la mejor de ellas.

La crítica de Sen al enfoque trascendental consiste en que sus criterios distributivos no son suficientes ni necesarios para orientar el camino de la construcción de una sociedad justa. En primer lugar, niega que los modelos idealizados puedan operar como criterios suficientes para ordenar distintas posibilidades en términos de su mayor o menor distancia de ese ideal. Sostiene que desde un modelo ideal no es posible tener acceso a las características que pueden ofrecer las posibles etapas intermedias, entre las que menciona: a) distintos campos de partida, b) variaciones en las dimensiones o transgresiones dentro del mismo campo general, y c) diferentes formas de evaluar las distintas infracciones a los criterios. Al considerar estas diferencias, Sen dice que la perspectiva de trascendencia no produce medios que permitan manejar este tipo de problemas, de tal forma que se alcance un ordenamiento que relacione, por ejemplo, los diferentes puntos de partida. Esto, en el contexto rawlsiano al que refiere explícitamente, puede significar que dados los distintos puntos de partida para satisfacer las demandas surgidas por el principio de la diferencia, se violen las exigencias de equidad de algunos de los afectados en la distribución de bienes primarios. Es decir, si tenemos diferentes demandas de personas que califican como miembros del grupo menos aventajado, como al principio de la diferencia no lo acompaña un criterio de aplicabilidad que permita satisfacerlas todas equitativamente, se correrá el riesgo de que durante el proceso de aplicación del principio la equidad se vea socavada.

Otro aspecto que Sen destaca es que también existen diferentes formas de evaluar el alcance de cada discrepancia que se presente y de apreciar la lejanía o cercanía de las distribuciones actuales con respecto a los principios de justicia. Sen habla de la necesidad de alcanzar algún tipo de equilibrio entre las posiciones, pero sostiene que este ejercicio interpretativo se encuentra más allá del vocabulario específico de los enfoques trascendentales que tienen su preocupación central en la identificación del momento ideal. "La caracterización de una justicia impoluta no conlleva ningún lineamiento acerca de cómo pueden compararse y ordenarse diversos puntos de partida desde lo perfecto" (Sen 2006, p. 220). ${ }^{1}$ De ahí que sostenga que quienes defienden una

\footnotetext{
${ }^{1}$ Todas las traducciones son mías.
} 
posición trascendental no perciben como un problema la imposibilidad de realizar el tipo de comparaciones indicadas.

En contrapartida, para Sen, la identificación de la "sociedad justa" es insignificante, incluso en términos de orientar los mejores arreglos sociales graduales, ya que tal identificación no especifica un ordenamiento único que conduzca a lo mejor, debido a que se puede llegar a lo mejor a través de diferentes ordenamientos. Como analogía para descartar la suficiencia del criterio del modelo ideal, Sen utiliza el ejemplo de que el hecho de considerar la Mona Lisa como la mejor pintura del mundo no revela cómo ordenar a Gauguin frente a Van Gogh. No obstante, es preciso señalar que Sen no percibe que en este caso sí podría preguntarse por qué considerar la Mona Lisa como la mejor pintura, y seguramente la respuesta revelaría un criterio que permitiese calificar a Gauguin y a Van Gogh. La identificación del modelo ideal que surgiría en el caso de la pregunta por la Mona Lisa, trasladada al caso de la justicia, posibilitaría identificar criterios normativos intermedios para hacer comparaciones de justicia y realizar ordenamientos como parte del camino hacia esa sociedad justa. Eso permitiría conjugar la identificación de un modelo ideal con la aplicabilidad que demanda tomar medidas intermedias para alcanzarlo; pero ésta es una posibilidad que Sen no admite.

Aún más, Sen también rechaza la hipótesis de que la identificación del mejor arreglo de justicia sea algo necesario aunque no suficiente para ordenar alternativas en términos de justicia. Al respecto dice que la evaluación relativa de dos alternativas es una cuestión entre ellas y que no requiere incorporar una tercera.

De hecho, no es en absoluto obvio por qué al juzgar que un arreglo social $x$ es mejor que un arreglo alternativo $y$ se deba invocar la identificación de que una muy diferente alternativa z es la "mejor" o "correcta". $\mathrm{Al}$ argumentar a favor de un Picasso en lugar de un Dalí no es necesario preocuparse por identificar la pintura más perfecta del mundo, que superaría a los Picasso y los Dalí y a todas las otras pinturas del mundo. (Sen 2009, p. 101)

Puede afirmarse en este caso que la tercera alternativa en juego permitiría averiguar el criterio que hace posible identificar en función de qué consideramos mejor una alternativa que otra. Los criterios para el ordenamiento no están especificados y eso es lo que una tercera opción, en cuanto que es la mejor, puede aportar; pero, para ello, lo que se debería indagar no es si es la mejor, sino por qué lo es. De nuevo, la pregunta 
de por qué una alternativa es la mejor devela el criterio normativo que está en juego para el procesamiento de las evaluaciones.

En resumen, Sen sostiene que las teorías de la justicia, en su intención de presentar un modelo ideal, no aportan un criterio suficiente para manejar los problemas que surgen de las diversas demandas de aplicabilidad, ya que no producen los medios apropiados para lograrlo. También sostiene que no son necesarias, porque la evaluación de dos alternativas no requiere la presencia de una tercera provista por alguna teoría de la justicia.

Si bien para Sen no es imposible que un modelo ideal conviva con la incompletud, y a esto lo denomina teorías "conglomeradas" (cfr. Sen 2009, p. 97), dicha posibilidad es marginal; en los casos de las teorías de la justicia que él considera, y entre las que destaca la justicia rawlsiana, tal posibilidad no existe. La razón de ello se encuentra en los rasgos que atribuye a las teorías de mayor impacto del modelo trascendental. Si se considera que este modelo da una respuesta de una vez y para siempre, con la cual establece todos los pasos intermedios y todos los ordenamientos para poder alcanzar ese estado ideal, entonces la irreconciliabilidad entre los modelos se sostiene.

Sin embargo, esta interpretación de lo que Sen denomina enfoque trascendental no se ajusta en forma suficiente a los candidatos que supuestamente lo ejemplificarían. La teoría de Rawls, que es a la que Sen refiere, no coincide con los rasgos totalistas que se le atribuyen. En la justicia como equidad es posible percibir la convivencia de la incompletud con la presentación de un modelo ideal; esto se manifiesta en que Rawls dirige su propuesta a lo que él denomina "sociedades democráticas" o "democracias constitucionales", que no coinciden con el modelo idealizado pero que podrían hacerlo (cfr. Rawls 1996, pp. 4142). Si bien, como sostiene Sen, Rawls no muestra explícitamente una especial preocupación por la realización efectiva de su modelo ideal, esto no bloquea la posibilidad de que la construcción de una sociedad justa esté orientada por dicho modelo, tal como Sen reconoce en el caso de las teorías "conglomeradas". Por otra parte, para descartar la interpretación que hace Sen de la justicia rawlsiana, es preciso recordar que, en "Unidad social y bienes primarios" (1986b), Rawls parece haberse anticipado a la posición de Sen y sostiene explícitamente que lo relevante en una teoría de justicia no consiste en "unos pocos principios universales aplicables a todos los casos. Lo que se requiere es que desde el punto de la posición original, o en algún otro estadio apropiado, toda la familia de principios pueda combinarse dentro de un marco coherente de deliberación" (Rawls 1986b, p. 195), y en nota a pie de página 
agrega que "los pesos del índice de bienes primarios no necesitan ser establecidos en la posición original de una vez por todas, y con todo detalle, para toda sociedad bien-ordenada".

Estas afirmaciones, al igual que los conceptos antes indicados, permiten concluir que la propuesta de Rawls no se ajusta adecuadamente a lo que Sen denomina enfoque trascendental, y más bien permite compatibilizar el enfoque trascendental y el comparativo a través de una serie de posibles etapas y ajustes en el camino hacia la realización de una sociedad justa.

Otro caso muy destacable en el debate sobre la justicia distributiva en el que se da la posibilidad de que convivan los enfoques que Sen llama trascendental y comparativo es el de la igualdad de recursos de Dworkin, un modelo de justicia ideal aplicable al mundo real a través de un conjunto de posibles candidatos. Estos candidatos, coincidentes conceptualmente con lo que Sen llama el enfoque comparativo, se denominan situaciones igualitarias defendibles (cfr. Dworkin 1987) y serán elegidos en función del criterio ideal.

Las situaciones igualitarias defendibles son aquellas que a través de medidas técnicamente posibles se acercan al ideal igualitario. Estas situaciones no son idénticas al ideal de la igualdad de recursos que Dworkin presenta, pero significan un acercamiento considerable al mismo, dadas las limitaciones técnicas que se sufrirían en caso de tener un compromiso absoluto con la justicia.

Lo mejor que podemos hacer ahora, por consiguiente, al identificar distribuciones defendibles para nuestra comunidad, es imaginar un conjunto de tales esquemas, siendo cada uno de ellos técnicamente posible a través de una serie de pasos que podríamos comenzar a tomar inmediatamente. Por eso hablaré de aquí en delante de una distribución defendible, significando con ello un miembro de tal conjunto, en lugar de la distribución defendible. (Dworkin 1987, p. 44)

En la busca de la igualdad hay un conjunto de situaciones igualitarias defendibles para nuestra comunidad que deben ser ordenadas para poder elegir, entre las posibles, la que mejor realiza el ideal igualitario defendido. Para ello, Dworkin se vale de su modelo distributivo como criterio de ordenación y, por lo tanto, las distribuciones defendibles deberían ser ordenadas de acuerdo a la factibilidad que puedan tener en nuestra comunidad, es decir, según la probabilidad de que nuestra comunidad alcance esa distribución si se ordenase en función de los parámetros distributivos del modelo ideal de Dworkin. 
La ordenación de las diferentes distribuciones defendibles que puedan reconocerse no es una ordenación completa según su factibilidad, porque "no tendríamos esperanza alguna de ser capaces de decidir, para cada par de ellas, si una es más convincente que la otra o si ambas lo son igualmente" (Dworkin 1987, p. 44). Sin embargo, se puede esperar razonablemente una ordenación incompleta en virtud de la cual podamos ser capaces de decidir cuáles distribuciones defendibles son más factibles que otras. En este punto, Dworkin defiende una posición idéntica a la de Sen (cfr. 1995, pp. 62-63) sobre la imposibilidad de alcanzar una ordenación completa, aunque hace convivir el modelo trascendental con el comparativo.

Tanto en el caso de Rawls como en el de Dworkin, la distancia entre el modelo idealizado y las medidas potenciales que conducirían a una sociedad justa depende de interpretaciones que toman en cuenta la tradición de la sociedad, sus características institucionales o la factibilidad de la implementación de ciertas medidas; esto no es ni más ni menos que un ejercicio interpretativo de aplicación que, además de no ser excluido por la formulación de situaciones idealizadas, es requerido por éstas. Es este ejercicio interpretativo lo que permite superar las dificultades que Sen indica, y las supera, por ejemplo, a través de la introducción de criterios normativos intermedios, de menor nivel de abstracción que los principios de justicia, que permiten reducir la incertidumbre inherente a toda interpretación. Esta incertidumbre es la que posibilita que la respuesta o el ordenamiento al que se arribe sea siempre revisable, y justamente en este punto es donde se asienta la dinámica del desarrollo de la práctica de la justicia (cfr. Dworkin 1996, pp. 129-137). En este camino de reducción de la incertidumbre y de la interpretación o reinterpretación de posibles respuestas a los requerimientos de la justicia es imprescindible contar con un criterio normativo que oriente la práctica interpretativa; por ello sostengo que tanto el modelo idealizado como los posibles criterios normativos intermedios son protagonistas inevitables en esta tarea.

Puede afirmarse que la posición de Sen está muy cerca de la caracterización del intuicionismo que hace Rawls, ya que para Sen, al igual que para el intuicionismo, a la hora de establecer una ordenación de las situaciones más justas existe un nivel de generalidad en el que no es posible encontrar un criterio que pueda reducir la incertidumbre (cfr. Rawls 1979, p. 53). Por lo tanto, no sólo no es posible proveer principios que cumplan con tal función, tampoco es posible apelar a un método o a reglas de prioridad que puedan reducir tal incertidumbre; esto deja a nuestras capacidades intuitivas como único recurso disponi- 
ble para solucionar la complejidad de la aplicabilidad, y si bien Sen no lo menciona explícitamente, creo que existe una apelación subyacente a ello.

A diferencia de Sen y en consonancia con Rawls y Dworkin, considero que una teoría de la justicia está constituida por dos momentos: uno ideal que establece las características de una sociedad justa, y uno de aplicabilidad en el que ese ideal es ajustado a las condiciones locales. En estos dos rasgos radica lo que denominé al inicio de este trabajo la primera faceta del alcance de una teoría de justicia, sin los cuales sería imposible contar con criterios normativos que permitan realizar un ordenamiento de alternativas, y tampoco se podría avanzar en una creciente adecuación de tales criterios a las demandas que imponen las circunstancias variables de aplicabilidad. Sin embargo, esto no opaca lo que considero el mayor aporte de Sen a la discusión sobre justicia distributiva: la introducción de una base de información altamente sensible a la variabilidad intersubjetiva. A continuación desarrollaré esto con mayor detalle y presentaré la posibilidad de que el enfoque de las capacidades desempeñe un papel mayor que el que Sen pretende en una teoría de justicia. En esta tarea tomaré como referencia la teoría de la justicia de Rawls y cómo puede ser expandida desde el enfoque de las capacidades.

\section{La crítica de Sen a la justicia rawlsiana}

El enfoque de las capacidades de Sen ha tenido como característica distintiva y aporte principal a la discusión sobre la justicia distributiva la introducción de un nuevo espacio focal para realizar las evaluaciones de justicia. Su intención ha sido proveer un marco normativo que permita realizar la evaluación del bien-estar de las personas. Para ello pretende superar los riesgos subjetivistas que puede tener la búsqueda de una concepción adecuada de este concepto, puesto que es posible que al categorizar aquello que es valioso para una persona se incluyan todas sus preferencias con igual valor; esto se da en el caso de las teorías bienestaristas que, al asignar un igual peso a las preferencias de las personas cualesquiera sean ellas, tienen como consecuencia que la preferencia de alguien por cenar en un restaurante caro y la de otra persona por satisfacer el hambre con un bocadillo en su casa sean igualmente relevantes (cfr. Sen 1979, pp. 470-471; 1994, pp. 146-148). Sen se aleja de este riesgo y pretende construir un criterio objetivo de bien-estar. En esta tarea se aparta de lo que denomina la rigidez de los medios, inherente a las propuestas de Rawls y Dworkin, las cuales, para 
evitar el subjetivismo bienestarista, se concentran en los medios como parámetro de justicia, y esto conlleva que no se perciba adecuadamente la relevancia de la variabilidad interpersonal para las cuestiones de justicia. Lo que Sen sostiene al respecto es que las bases de información de los bienes primarios de Rawls y de los recursos de Dworkin son rígidas con respecto a cómo varían las capacidades de las personas; por esto pueden llegar a estipular que dos personas son tratadas igualitariamente si tienen el mismo conjunto equitativo de bienes primarios o recursos, lo que oculta el hecho de que ambas personas pueden diferir sustancialmente en sus capacidades de transformar esos medios en bienestar (cfr. Sen 1994, pp. 149-153).

Luego de alejarse de estas dificultades, Sen presenta como espacio para realizar las evaluaciones de justicia el de las capacidades; esto supone poner el énfasis ya no en las políticas distributivas de medios como los bienes primarios o simplemente el ingreso, sino en lo que éstos significan para los seres humanos. La diferencia radical que existe entre las distintas personas vuelve necesario tal movimiento, ya que la conversión de medios en bienestar varía sustancialmente de una persona a otra, y tal variación se explica mediante el concepto de capacidad; es decir, las personas transforman los mismos medios en diferentes logros sólo porque difieren en el desarrollo de sus capacidades.

Partiendo de esto, Sen sostiene que la vida puede concebirse como un conjunto de funcionamientos o logros; esto es, un conjunto de estados y acciones en el que la realización de una persona puede entenderse como el vector de sus funcionamientos, y éstos abarcan cosas como "estar bien alimentado", "tener buena salud" o "participar en la vida de la comunidad". Los funcionamientos son constitutivos del estado de una persona, y la evaluación del bien-estar depende de la evaluación de estos elementos. Por su parte, la idea de capacidad representa las diversas combinaciones de funcionamientos que puede realizar una persona; de ahí que la capacidad de una persona refleje su libertad para llevar un tipo de vida u otro (cfr. Sen 1995, pp. 53-54). Es en función de esto como las capacidades pueden actualizarse a través de un haz de funcionamientos, y queda a discreción del sujeto la posibilidad de tal actualización; la libertad de una persona, en tal sentido, se encuentra bajo el alcance de esa potencialidad que tienen las capacidades de realizarse por medio de diferentes funcionamientos.

La crítica de Sen a la rigidez de medios es una de las más importantes que ha sufrido la justicia como equidad, aunque es preciso decir que no ha tenido un gran impacto en la posición de Rawls, quien la consideró como una contribución que se debe tener en cuenta en 
las etapas posteriores a la posición original, es decir, en un momento en que la aplicabilidad requiere manejar más información para tomar decisiones coincidentes con la justicia (etapa legislativa) y en la que una noción más comprehensiva que la de los bienes primarios puede ser de suma utilidad (cfr. Rawls 1986b, p. 195, n. 8). Por otra parte, la descripción que Rawls ha hecho de los bienes primarios toma en cuenta las capacidades elementales de las que habla Sen, en cuanto capacidades de la personalidad moral que determinan la condición de ciudadanos como personas libres e iguales. Esto es así porque el índice de bienes primarios se construye a partir de preguntar qué cosas son necesarias para que los ciudadanos puedan ejercer sus capacidades de la personalidad moral, mantengan su condición de personas libres e iguales y sean miembros plenamente cooperativos de la sociedad (cfr. Rawls 2002, pp. 224-225).

Recordemos que al concepto de persona moral lo especifican las dos capacidades morales que sustentan la libertad e igualdad de todo miembro plenamente cooperante de la sociedad, que es la forma de Rawls de concebir a los ciudadanos. Estas dos capacidades morales son la del sentido de justicia y la capacidad para tener una concepción del bien. El sentido de justicia consiste en entender, aplicar y actuar de acuerdo a una concepción pública de justicia que tiene como característica el definir los términos equitativos de la cooperación social. Por su parte, la capacidad para concebir el bien consiste en formar, revisar y perseguir racionalmente una concepción de la propia ventaja o del propio bien referido a lo que es valioso en la vida humana. Estas facultades se corresponden con los intereses de orden supremo de realizar y ejercer esas facultades. A su vez, las personas morales poseen un tercer interés que mueve a las partes, y éste es "un interés de orden superior en proteger y promover su concepción del bien, cualquiera que pueda ser, de la mejor forma posible" (Rawls 1986a, p. 146). Rawls sostiene que estas capacidades abarcan el concepto de capacidades de Sen, por lo que la relación entre bienes primarios y capacidades cuestionada por la crítica de la rigidez de los medios sí estaría considerada en la justicia como equidad (cfr. Rawls 2002, p. 225). Si bien esta posición -que puede considerarse como la última respuesta de Rawls a la crítica de la rigidez- salva el obstáculo, es preciso señalar que el concepto de capacidades elementales de Sen es más comprehensivo que el de las capacidades de la personalidad moral, por lo que no son fácilmente equiparables. De todas formas, lo que puede constatarse es que la crítica de Sen no ha afectado de manera considerable a la justicia rawlsiana. 
Sin embargo, lo que resulta realmente desestabilizante para la teoría de Rawls es que el foco de la polémica se traslade de la teoría ideal a la aplicabilidad en las sociedades democráticas. Como se ha señalado, puede reconstruirse la convivencia de un momento ideal y otro de aplicabilidad en la teoría de la justicia de Rawls, aunque es preciso reconocer que no manifiesta una especial preocupación por ello. Esto lo señala especialmente Sen, quien pone particular énfasis en la dificultad que significa para la realización de una teoría de la justicia el que los miembros de una sociedad no sean razonables (2009, p. 79); para mostrar tal dificultad pone especial atención en una serie de circunstancias relevantes para realizar la idea de justicia, como la pobreza o la marginación, que socavarían las capacidades elementales que permiten alcanzar la condición de miembros plenamente cooperantes. Estas circunstancias establecen una diferencia radical en el desarrollo de las capacidades que permiten a una persona convertir medios en bienestar; es en estos casos donde la crítica de Sen se vuelve relevante, es decir, cuando nos preguntamos por las medidas que deberían tomarse para avanzar en la realización del ideal de justicia.

En ese movimiento hacia la aplicabilidad, la idealización de la persona moral de Rawls tiene suficiente potencial normativo como para ir más allá del modelo ideal y operar como criterio normativo que establezca lo necesario para que alguien pueda ser un miembro plenamente cooperante en las sociedades democráticas. De esta forma, la justicia rawlsiana, a pesar de lo que sostiene Sen, contribuiría con un criterio normativo intermedio, surgido del modelo ideal, para la realización de una sociedad justa, es decir, habría convivencia de idealización y aplicabilidad. Esta proyección va más allá de lo explícitamente sostenido por Rawls y pretende darle un mayor alcance a su propuesta, de tal forma que pueda cumplir con los requerimientos de justicia de las sociedades democráticas, y muy especialmente de aquellas sociedades contemporáneas donde las circunstancias que indica Sen —de pobreza, marginación y déficit en el desarrollo de capacidades elementalestienen una relevancia enorme.

El enfoque de Sen, por tener una preocupación central en la realización de la justicia, parece ser más versátil que la justicia rawlsiana a la hora de atender a las demandas de justicia de las sociedades más pobres. La convergencia que, como ya se indicó, Rawls reconoce entre las capacidades de la personalidad moral y las capacidades elementales de Sen, y la postulación de la idealización de la persona moral como criterio normativo que permita orientar la aplicabilidad permitirían conformar una estrategia de aplicabilidad apropiada para las sociedades 
democráticas al contar con la posibilidad de tomar en consideración no sólo a quienes por su desarrollo de capacidades pueden ser miembros plenamente cooperantes, sino también a quienes para lograrlo requieren la intervención específica de las instituciones sociales.

Una auténtica preocupación por la justicia debería postular una teoría de justicia con suficiente alcance como para considerar a los países con un porcentaje significativo de población pobre, y para ello debería postularse un principio de justicia que asegure universalmente que el ejercicio de las capacidades de la personalidad moral permita a las personas ser miembros plenamente cooperantes. Esta proyección de aplicabilidad está fuera del alcance de la teoría de Rawls y de la propuesta de Sen; la razón de ello es que, por una parte, introduce una preocupación por la aplicabilidad que es marginal en la justicia rawlsiana, y por otra, a diferencia de Sen, pretende introducir principios de justicia y criterios normativos para guiar la aplicabilidad. Sin embargo, esta tarea de presentar una teoría de la justicia de mayor alcance que la rawlsiana no puede prescindir de las virtudes que tiene el programa de Rawls, ya que, en su proyección hacia la aplicabilidad, el modelo ideal provee principios y criterios normativos que pueden orientar el diseño e implementación de los arreglos institucionales, y tampoco puede dejar de incorporar una base de información como la de las capacidades, que muestra ser más sensible a la vulnerabilidad que la de la justicia como equidad. Esta conjunción de virtudes y limitaciones de las propuestas de Rawls y Sen, al ser articulada adecuadamente, demandará que se traduzca en un principio de justicia que tenga por objeto incluir a los sujetos de las sociedades democráticas que, por no tener un adecuado desarrollo de capacidades, no son plenamente cooperantes. A la vez, será posible especificar el umbral de ser plenamente cooperante mediante un conjunto de capacidades que incluya las de la personalidad moral, pero que además integre las capacidades elementales sin las cuales es imposible alcanzar la personalidad moral, como la capacidad de estar bien alimentado, de tener una vivienda digna o de estar libre de enfermedades. Este paso dotaría a la justicia como equidad de Rawls de un alcance mayor que el que hoy tiene, y le permitirá proyectarse hacia la realización del ideal de justicia en las sociedades democráticas. Tal expansión es lo que denominé, al inicio de este artículo, la segunda faceta del alcance de una teoría de la justicia, y que desarrollaré a continuación. 


\section{La ampliación de la justicia rawlsiana}

Como ya se indicó, Rawls mismo reconoce la posibilidad de que su propuesta se complemente con las capacidades elementales de Sen; pero es preciso agregar que la posibilidad de un encuentro entre ambas perspectivas se ve reforzada por los planteamientos de Martha Nussbaum, quien en su influyente versión del enfoque de las capacidades incorpora la condición de persona moral como criterio normativo para identificar y establecer todo lo que las instituciones de la sociedad deberían asegurar a una persona (cfr. Nussbaum 2000, pp. 64-68).

Mi posición, como ya he adelantado, consiste en que un programa de justicia distributiva que pretenda responder a los requerimientos de las sociedades más pobres deberá garantizar que todos puedan alcanzar efectivamente la condición de miembros plenamente cooperantes; para ello, las capacidades de la personalidad moral deberán desarrollarse hasta el umbral de dicha condición.

Una consideración no exhaustiva de lo requerido para el ejercicio mínimo de las capacidades de la personalidad moral indica que, para albergar una concepción del bien, revisarla y llevarla adelante, será necesario identificar alternativas, evaluarlas, priorizarlas y en función de ello decidir cuál es el mejor curso de acción. Para hacer una revisión crítica de las propias posiciones también es imprescindible lograr un cierto distanciamiento, lo cual se logra a través de un ejercicio autorreflexivo en el que es posible cuestionar las propias tradiciones, los patrones de comportamiento que nos afectan, así como nuestras creencias acerca de lo que es una vida buena o un proyecto vital valioso. Ambos aspectos, elección racional y autorreflexión, son rasgos claramente distintivos de la racionalidad práctica que ha de ser desarrollada. A su vez, para poder realizar las actividades indicadas en este proceso es necesario garantizar que las personas tengan plena confianza en su capacidad para llevar adelante un proyecto vital y que se sientan igualmente respetadas e igualmente consideradas por otros; sin estas precondiciones es prácticamente imposible que perciban como valiosos los planes vitales que llevarán adelante, o que experimenten como igualmente valiosas las alternativas que puedan presentar a dichos planes. Rawls considera este aspecto en especial al incluir entre los bienes primarios las bases sociales del autorrespeto.

De acuerdo con lo anterior, para alcanzar un desarrollo mínimo de la capacidad para albergar una concepción del bien, por lo menos tendrían que garantizarse capacidades que permitiesen ejercer el razonamiento lógico formal, a la vez que el razonamiento práctico; pero esto 
no es suficiente, porque las personas también deben ser capaces de usar la imaginación y los sentidos de tal forma que la racionalidad adquirida sea lo suficientemente amplia como para que las emociones tomen parte en la constitución de los planes vitales y en la priorización de alternativas. Un camino posible para fomentar el desarrollo de estas capacidades es a través de programas educativos en los que se trascienda la herencia positivista que afecta a buena parte de las sociedades contemporáneas, por la cual lo central de la educación es la alfabetización y el entrenamiento científico y matemático, pero que asigna a las humanidades un papel secundario o marginal. Las humanidades, como la filosofía y la literatura, son justamente las que contribuyen significativamente a desarrollar el pensamiento crítico capaz de realizar procesos autorreflexivos o de generar vínculos empáticos que permiten ampliar el campo de reconocimiento del otro, y sin los cuales el ejercicio de la racionalidad práctica se vería considerablemente disminuido. Por último, solamente una educación en la que las emociones no sean consideradas como la antítesis de la razón, sino como su aliado principal, podrá contribuir a garantizar la propia valía, y cada cual se sentirá capaz de manifestar sus propias opiniones y hacerlas pesar en los contextos apropiados.

Por su parte, la capacidad de la personalidad moral del sentido de justicia demanda que las personas lleguen a un acuerdo no sólo en cuanto a los beneficios que tiene la cooperación social, sino también con respecto a sus cargas. El requerimiento de entender y aplicar una concepción pública de la justicia demanda potenciar la capacidad de ponerse en el lugar del otro y de esa forma comprender tanto los potenciales beneficios como las necesarias cargas que deben asumirse. Para ello, el contar con un desarrollo de sentimientos empáticos puede ser un camino apropiado, al igual que el enfatizar el reconocimiento de la propia vulnerabilidad. La literatura, por ejemplo, es y ha sido un medio idóneo para ello, debido a que las narraciones tienen como rasgo distintivo el ampliar el mundo del lector y permitirle, a través de la imaginación, entrar en el de los protagonistas (cfr. Pereira y Modzelewski 2006, pp. 116-119; Nussbaum 1997, pp. 85-86, y 2005, pp. 238-248). De esta forma, el lector experimenta universos que de otra forma le serían absolutamente ajenos y puede sentir, a través del mundo del texto, cosas como la pobreza, la sumisión de la mujer, el ser víctima de la violencia o la discriminación racial o sexual. Por lo tanto, empatía y vulnerabilidad son dos componentes centrales para propiciar la autocomprensión de las personas como seres necesitados, frágiles y vulnerables, capaces de reconocer al otro también como un ser frágil y necesitado, 
a la vez que capaz de cooperar en lo social. Esto implica que las personas asuman la perspectiva del otro a partir de la internalización de las expectativas normativas de sus compañeros de interacción (cfr. Mead 1934), y es de esta forma como podemos sabernos reconocidos como miembros de la cooperación social.

Estas capacidades, si bien son determinantes para la condición de persona moral, necesitan ser complementadas con otras que contribuyen igualmente a garantizar la dignidad y un sentido de la propia valía, y en virtud de ello sería necesario erradicar todas aquellas circunstancias que afecten la posibilidad de autocomprenderse como alguien considerado igualitariamente. Pensando en algunos de los ejemplos que Sen ha brindado, podría pensarse sin mayor dificultad en la capacidad de estar libre de enfermedades, de contar con una vivienda digna, de estar bien nutrido, de aparecer en público sin sentirse avergonzado, etc. Una vez que estas capacidades se encuentren desarrolladas mínimamente, las personas alcanzarán la condición de miembros plenamente cooperantes.

Probablemente la generación de preferencias adaptativas es una de las situaciones que ilustra muy bien la incidencia del déficit de desarrollo de capacidades en situaciones como la pobreza extrema o la violencia doméstica. En estos casos, muchas veces nos preguntamos por qué las personas afectadas por tales circunstancias no toman algunas decisiones para revertir su situación. Una posible explicación es el desarrollo de preferencias adaptativas, las cuales son un tipo de preferencias resultado de un proceso por el cual se da el ajuste de las voliciones a las posibilidades del afectado; esta adaptación se manifiesta como una tendencia a eludir la frustración que se siente al experimentar voliciones que no pueden satisfacerse (Elster 1988, p. 42). El resultado es que en situaciones de suma precariedad, como la pobreza extrema o la violencia doméstica, las personas pueden generar una evaluación positiva acerca de su situación y manifestar una alta conformidad con ella. La mayor probabilidad de que se presenten estas preferencias surge en casos donde, al decir de Elster, las personas son menos autónomas, y creo que sin mayor dificultad se pueden introducir, como capacidades clave para contrarrestar la influencia de las preferencias adaptativas, la autorreflexión y la capacidad para acordar con otros. Como ya se señaló, a partir de estas capacidades es posible que las personas generen un distanciamiento de las propias situaciones y, a su vez, tengan una apertura a los otros que redelimite sus horizontes vitales, lo cual les permita integrar elementos cognitivos que disparen la autorreflexión y los ponga en condiciones de eliminar tales preferencias. 
A partir de estas consideraciones puede afirmarse que el objetivo de expandir una propuesta de justicia distributiva para tener en cuenta las sociedades más pobres permite la formulación de un principio de justicia que considere dos aspectos: por una parte, que sea lo suficientemente sensible a las circunstancias que obstaculizan el logro de la condición de miembros plenamente cooperantes, y, por otra, que tome las capacidades como base de información. El asumir las capacidades como base de información hace que las posibles medidas para solucionar situaciones de injusticia deban explicarse, en última instancia, en términos de un desarrollo o expansión de capacidades elementales. Por ello, la toma de posibles medidas, por ejemplo, para garantizar oportunidades o para incrementar el ingreso de algunos grupos, estará justificada siempre y cuando con ellas se desarrollen las capacidades elementales que permitan asegurar la condición de miembros plenamente cooperantes. El principio mencionado diría lo siguiente:

Todas las personas deben tener asegurado, durante toda su vida, por lo menos un desarrollo mínimo de capacidades elementales que les permita ser miembros plenamente cooperantes de la sociedad.

El principal aporte que pretendo hacer a través de la introducción de este principio es expandir el alcance de una teoría de la justicia para que pueda tener como campo de aplicación las sociedades democráticas $y$, en especial, aquellas que se encuentran severamente afectadas por la desigualdad social, la pobreza y la marginación social. Por ello debe tomarse la postulación del principio sólo como una manifestación de la expansión del alcance de un programa de justicia social, pero de ninguna manera como el fin mismo de tal expansión. En virtud de ello, el principio es falible y estará sujeto a revisión y ajuste cuando las circunstancias a las que se aplique obliguen a hacer una formulación diferente que posibilite una mejor realización del ideal de justicia. Ésta es la dinámica que defendí en la primera parte de este trabajo, en la que los principios son sensibles a las condiciones de aplicabilidad y se los ajusta cuando ellas así lo requieran.

\section{Una lista de capacidades como guía de la aplicabilidad}

Volviendo a la formulación del principio, surge ahí la pregunta de si es posible elaborar una lista de capacidades elementales que opere como 
guía para evaluar la aplicabilidad de las medidas que deberán asegurar la condición de miembros cooperantes de la sociedad. La utilidad de esta lista radicaría en que, a partir de ella, se podría identificar todo lo necesario para que alguien alcance el desarrollo de sus capacidades de la personalidad moral que le permita ser racional y razonable, y a la vez posibilitaría establecer un umbral que estipularía cuándo alguien efectivamente ha alcanzado tal condición. En consecuencia, a partir de esta lista sería posible, por una parte, evaluar la situación de una persona en cuanto a si se encuentra más cerca o más lejos del umbral, y, por otra, se podrían promover políticas sociales orientadas a garantizar que las personas efectivamente superen tal umbral.

La lista de capacidades que Nussbaum introduce es la que más se ajusta a la función indicada y, además, coincide con las consideraciones hechas sobre la necesidad de complementar las capacidades de la personalidad moral con otras capacidades que propiciarían el desarrollo de las primeras. La razón para tomar la lista de Nussbaum es que, como ya se señaló, ella parte de la idealización del sujeto de la persona moral rawlsiana ( $c f r$. Nussbaum 2000, p. 119) y ésta es una coincidencia muy importante con la perspectiva desarrollada en este trabajo. En su construcción de la lista, Nussbaum parte de un conjunto de capacidades que tendrían como característica el ser determinantes de la vida humana, es decir, aquellas sin las cuales un ser humano dejaría de serlo.

El objetivo de Nussbaum es fundar un criterio normativo fuertemente universalista, y para ello parte de dos hechos: el primero es que con base en algunas características específicas se puede reconocer a los otros como seres humanos a lo largo de distintas coordenadas de espacio y tiempo, y el segundo es que existe un consenso general acerca de las características cuya ausencia significaría el fin de una forma de vida humana (cfr. Nussbaum 2000, pp. 73-75).

Estos hechos ponen las bases para la elaboración de una lista abierta de características que hacen la condición humana. Las capacidades a las que se refiere Nussbaum son las que permiten a un individuo llevar una vida digna. Por lo tanto, como estamos hablando de las capacidades mínimas que determinan la condición de persona moral y estos mínimos son los exigibles por la dignidad humana, creo que es más apropiado hablar de mínimos de dignidad (cfr. Pereira 2007, pp. 35 y ss.). La lista de capacidades incluye algunas tan básicas como "ser capaz de tener buena salud", "de estar bien alimentado", "de tener una vivienda adecuada" o "ser capaz de asegurar la propia integridad física", hasta capacidades más complejas como "ser capaz de imaginar, de pensar y de usar la razón", "ser capaz de formar una concepción del bien y 
reflexionar críticamente acerca de la planificación de nuestra vida" o "ser capaz de participar efectivamente en las elecciones que gobiernan nuestra propia vida" (Nussbaum 2000, pp. 78-80).

La lista de Nussbaum podría operar, entonces, como guía normativa para implementar medidas institucionales y diseñar políticas sociales, de forma que se asegure a todas las personas, durante toda su vida, un desarrollo mínimo de capacidades elementales que les permita ser miembros plenamente cooperantes de la sociedad.

En este momento, y recordando lo que se ha planteado en el primer apartado de este trabajo, es preciso decir que Sen, además de oponerse a la posibilidad de presentar principios de justicia, también rechaza con argumentos similares la posibilidad de construir una lista de capacidades elementales. Con respecto a la lista de capacidades, sostiene la imposibilidad metodológica de una ordenación completa. Según Sen, su opción por el uso de la alternativa de una ordenación parcial se sustenta en dos tipos de razones; la primera de ellas es que la idea de desigualdad presenta cierta ambigüedad, lo que determinaría que un intento de ordenación completa, al basarse en un concepto ambiguo, fuese erróneo. La segunda razón es que "aunque no fuera un error buscar una ordenación completa, en la práctica podemos no ser capaces de identificarla" (Sen 1995, pp. 62-63). Pero si bien tanto la posibilidad del error como la de la propia falibilidad desaconsejan una ordenación completa, Sen sostiene que es perfectamente posible hacer un ordenamiento parcial que posibilite la jerarquización de algunas situaciones sobre otras.

Éstas son las razones metodológicas de las que Sen se vale para rechazar la lista de Nussbaum, pues, a pesar de que él reconoce que las capacidades tienen una variación intercomunitaria significativamente menor a la de los requerimientos de medios para su ejercicio, considera que una lista fija con pretensiones de universalidad es tan específica que reduce las posibilidades de ajuste local, socavando la sensibilidad a la variación intercomunitaria, la cual es una de las consecuencias más valiosas de la incompletud del ordenamiento. De hecho, tanto la potencialidad como la utilidad del enfoque de las capacidades se encuentran respaldadas en una importante distinción conceptual entre la generalidad del enfoque y la particularidad de un listado específico de funcionamientos que apunta a salvaguardar dicha variación intercomunitaria (cfr. Sen 1993, p. 47).

Sin embargo, es necesario reconocer, en defensa de Nussbaum, que sus trabajos tienen como objetivo principal asegurar la sensibilidad a la variabilidad intercomunitaria. Esto último es un rasgo distintivo del 
concepto de capacidad, ya que posibilita que un mismo conjunto de capacidades elementales pueda especificarse en forma sustancialmente diferente en función de las condiciones locales, variando tanto el peso interno de cada capacidad como el nivel de desarrollo de las mismas. Es más, este desarrollo particular del enfoque de las capacidades que realiza Nussbaum permite superar cualquier sospecha de perfeccionismo que se le pueda imputar desde una perspectiva liberal (cfr. Rawls 1996, pp. 216-220), ya que aquello que debe ser objeto de las políticas públicas no es un listado de funcionamientos que especificarían cierta idea de buena vida, sino el conjunto de capacidades que colocan a todas las personas en condiciones de poder elegir y realizar un plan de vida (cfr. Nussbaum 2006, pp. 171-173; 2000, pp. 87-90).

Una vez que el concepto de capacidad adquiere el carácter transcultural y transcomunitario que Nussbaum le impone, y que se especifica comunitaria y culturalmente a través de un conjunto de funcionamientos, la crítica de Sen se diluye y es tan inocua como la crítica a los modelos trascendentales de justicia. Por lo tanto, el principio de justicia que se ha presentado y que pretende expandir el alcance de una teoría de la justicia, al garantizar universalmente el desarrollo mínimo de las capacidades elementales que permitan alcanzar la condición de miembros plenamente cooperantes, tendrá como guía para su implementación la lista de capacidades de Nussbaum.

Recordemos que la evaluación de los argumentos sobre las teorías de justicia de Sen nos condujo, en primer lugar, a rechazar su distinción entre modelos trascendentales y comparativos para sostener la posibilidad y necesidad de convivencia de ambas estrategias, con el fin de asegurar una sociedad justa; esto fue denominado la primera faceta del alcance de una teoría de la justicia. En segundo lugar, la base de información de las capacidades mostró ser de suma utilidad una vez que se ha proyectado una concepción de justicia como la rawlsiana hacia cuestiones de aplicabilidad, y a partir de ello se pueden reconocer circunstancias tales como un desarrollo deficitario de capacidades elementales. En tercer lugar, este movimiento hacia la realización del ideal de justicia hizo posible postular una propuesta aplicable a los países con alta incidencia de pobreza, y ello constituye la segunda faceta del alcance de una teoría de la justicia. Esto permitió introducir un principio que garantiza el desarrollo mínimo de capacidades elementales hasta el umbral que asegura la condición de miembro plenamente cooperante de la sociedad. Por último, estas capacidades pueden ser especificadas mediante una lista que funciona como guía normativa para la evaluación e implementación de políticas públicas. 
En este momento cabe preguntarse si una vez superado el umbral que garantiza la condición de miembros plenamente cooperantes, la base de información será la misma, y si será necesario introducir una nueva formulación de un principio de justicia.

\section{Capacidades y medios}

Si el principio que se ha presentado en el apartado anterior se aplicase exitosamente y las personas efectivamente lograran un desarrollo adecuado de sus capacidades de la personalidad moral, entonces las condiciones que hicieron necesario ese principio ya no se darían, y en cambio estaríamos bajo las condiciones supuestas por la justicia como equidad de Rawls. En este momento, que es una segunda instancia en la realización del ideal de justicia, ya contamos con sujetos que califican como miembros plenamente cooperantes, y por ello se debe considerar si la base de información de las capacidades sigue siendo la apropiada o si será necesario utilizar los bienes primarios como criterio para las comparaciones interpersonales.

Por debajo del umbral que asegura la condición de miembros plenamente cooperantes, la fragilidad ante las circunstancias bloquea tanto la posibilidad de responsabilizar en términos absolutos a las personas por su toma de decisiones, como la de plantear su contribución a la cooperación social. Además de injusto, sería cínico responsabilizar plenamente a alguien que haya nacido y se hubiese desarrollado en un contexto de pobreza extrema por la situación que vive; probablemente las decisiones que lo condujeron a esa situación reflejan un desarrollo de capacidades inadecuado para poder decidir libremente sobre su vida. Tampoco tendría mucho sentido pensar en la capacidad contributiva de personas que apenas cuentan con lo mínimo para poder sobrevivir. En estas condiciones, el imperativo que domina es el de garantizar el desarrollo de capacidades elementales orientadas a asegurar la condición de personas racionales y razonables.

La superación de este umbral implica que, si al poner en práctica el principio de justicia invocado se eliminan las condiciones que comprometían el desarrollo de capacidades del sujeto, se habrá incidido directamente en la vulnerabilidad del sujeto. Una vez que alguien califica como plenamente cooperante, significa que es capaz de compartir las cargas y los beneficios de la cooperación social, y en virtud de ello deberá contribuir a ella y deberá ser responsabilizado por los resultados de las decisiones que tome. La mayor vulnerabilidad que condujo a contar con una base de información de capacidades no podía plan- 
tear estos problemas. Una vez que se logran los mínimos requeridos para ser un miembro plenamente cooperante de la sociedad, tanto la responsabilidad por los resultados como la cooperación social deberán tematizarse. A partir de este momento, la justicia rawlsiana se muestra mucho más cercana a los requerimientos de aplicabilidad, puesto que cuenta con una base de información de medios que le permite incorporar a su dinámica distributiva y compensatoria tanto la responsabilidad por los resultados, como la capacidad contributiva de los afectados, aspectos que son problemáticos para la base de información de las capacidades.

Estas últimas consideraciones configuran la tercera faceta del alcance de una teoría de justicia, que también puede entenderse como una extensión de la segunda. Recordemos que la segunda faceta consistía en la expansión de una propuesta de justicia distributiva desde su formulación ideal hacia lo que demanda la realización de dicho ideal, enfocándose especialmente en las sociedades severamente afectadas por las desigualdades sociales. Una vez realizada tal expansión, la tercera faceta del alcance consiste en la articulación de dos bases de información: una de capacidades y otra de medios. Cada base de información impone características distributivas y compensatorias diferentes. La base de información de las capacidades requiere la operación del principio de justicia de capacidades ya presentado y que es necesario en los casos de las personas que se encuentren por debajo del umbral de miembro plenamente cooperante, mientras que una vez superado dicho umbral operaría una lógica distributiva y compensatoria de medios. ${ }^{2}$ Esta última implicaría el funcionamiento de la justicia rawlsiana con sus principios de justicia, que suponen la responsabilidad por las propias decisiones, la contribución a la cooperación social y la compensación en caso de que se presenten circunstancias desfavorables. Esta estrategia de desarrollar un programa de justicia distributiva de doble base de información tiene la virtud de potenciar la justicia rawlsiana en una dimensión de aplicabilidad y de proyectarla a sociedades con alta incidencia de pobreza y marginación social. ${ }^{3}$ Su expansión permite

${ }^{2}$ Es posible la construcción de un índice que permita discriminar diferentes grados de desarrollo de las capacidades de la personalidad moral; este trabajo se ha emprendido en las investigaciones del grupo "Ética, justicia y economía" de la Universidad de la República (cfr. Pereira y Vigorito 2010).

${ }^{3}$ La postulación de una teoría de justicia de doble base de información podría incorporar, en sustitución de la justicia como equidad, otros programas que compartieran sus rasgos distintivos principales, como la igualdad de recursos de Dworkin (cfr. Pereira 2004). Abordar una discusión sobre las virtudes de una u otra pers-

Diánoia, vol. LV, no. 65 (Noviembre 2010). 
una mejora tanto en el potencial explicativo como en la aplicabilidad de la justicia, a la vez que posibilita responsabilizar a los afectados por los resultados de sus decisiones, compensarlos por resultados adversos, consecuencia de circunstancias arbitrarias, e incorporarlos como contribuyentes a la cooperación social.

En conclusión, tanto la perspectiva de Sen como la de Rawls no son en sí mismas suficientes para proveer lo que las sociedades contemporáneas demandan de la justicia; las tres facetas del alcance de una teoría de justicia que he indicado pautan tales insuficiencias. La posición de Sen es incapaz de percibir la compatibilidad entre idealización y aplicabilidad en una teoría de justicia, y la perspectiva de Rawls no manifiesta una gran preocupación por la realización de su modelo. Ambas posiciones, sin embargo, pueden trabajar conjuntamente una vez que se pretende dar respuesta a sociedades donde la pobreza y la exclusión social tienen un protagonismo significativo. Sen puede proveer la base de información necesaria para garantizar el desarrollo adecuado de las capacidades de la personalidad moral, y Rawls puede suministrar la base de información apropiada y los principios de justicia una vez que se haya alcanzado la condición de miembro plenamente cooperante. De esta forma, mi defensa de un enfoque mixto con dos bases de información y sus correspondientes principios tiene la ventaja de aprovechar los aportes más significativos de las propuestas de Rawls y Sen, a la vez que supera las dificultades y bloqueos que ellas enfrentan. El resultado de esta tarea es una propuesta que abarca las tres facetas del alcance de una teoría de la justicia y que se preocupa por realizar la justicia de la mejor forma en la mayoría de las sociedades contemporáneas.

\section{BIBLIOGRAFÍA}

Dworkin, R., 1996, “Objectivity and Truth: You'd Better Believe It”, Philosophy and Public Affairs, vol. 25, no. 2, pp. 87-139.

_ 1987, "What Is Equality? Part III: The Place of Liberty", Iowa Law Review, no. 73 , pp. 1-54.

Elster, J., 1988, Uvas amargas. Sobre la subversión de la racionalidad, trad. Enrique Lynch, Península, Barcelona.

Mead, G.H., 1934, Mind, Self, and Society, The University of Chicago Press, Chicago.

Nussbaum, M., 2006, Frontiers of Justice, The Belknap Press of Harvard University Press, Cambridge, Mass.

—_, 2005, Upheavals of Thought, Cambridge, Cambridge University Press.

pectiva, o de las posibles reformulaciones de ambas, excede las intenciones de este trabajo. 
Nussbaum, M., 2000, Women and Human Development: The Capabilities Approach, Cambridge University Press, Cambridge.

—_ 1997, Justicia poética, trad. Carlos Gardini, Andrés Bello, Barcelona, 1997.

Pereira, G., 2007, ¿Condenados a la desigualdad extrema?, Centro de Estudios Filosóficos, Políticos y Sociales Vicente Lombardo Toledano, México.

—, 2004, Medios, capacidades y justicia distributiva, Instituto de Investigaciones Filosóficas-UnAM, México.

Pereira, G. y H. Modzelewski, 2006, "Ética, literatura y educación ciudadana para un mundo global", Isegoría, no. 34, pp. 111-128.

Pereira, G. y A. Vigorito (comps.), 2010, Preferencias adaptativas, autonomía y adaptación. Informe sobre la incidencia de las preferencias adaptativas en las políticas sociales, Fin de Siglo, Montevideo.

Rawls, J., 2002, La justicia como equidad. Una reformulación, trad. Andrés de Francisco, Paidós, Barcelona.

__ 1996, El liberalismo político, trad. Antoni Doménech, Crítica, Barcelona. -, 1986a, "El constructivismo kantiano en la teoría moral", en Justicia como equidad. Materiales para una teoría de la justicia, trad. Miguel Ángel Rodilla, Tecnos, Madrid, pp. 137-186.

__ 1986b, "Unidad social y bienes primarios", en Justicia como equidad. Materiales para una teoría de la justicia, pp. 187-211.

—_, 1979, Teoría de la justicia, trad. María Dolores González, Fondo de Cultura Económica, México.

Sen, A., 2009, The Idea of Justice, The Belknap Press of Harvard University Press, Cambridge, Mass. [Versión en castellano: La idea de la justicia, trad. Hernando Valencia Villa, Taurus, Madrid, 2010.]

— 2006, "What Do We Want from a Theory of Justice?", The Journal of Philosophy, vol. 103, no. 5, 2006, pp. 215-238.

_ 1995, Nuevo examen de la desigualdad, trad. Ana María Bravo, Alianza, Madrid.

—_, 1994, “iIgualdad de qué?”, en Libertad, igualdad y derecho, trad. Guillermo Valverde Gefall, Planeta-Agostini, Barcelona, pp. 133-156.

_ 1993 , "Capability and Well-Being", en A. Sen y M. Nussbaum, The Quality of Life, Clarendon, Oxford, pp. 30-53.

— 1979, "Utilitarianism and Welfarism", The Journal of Philosophy, vol. 76, no. 9, pp. 463-489.

Recibido el 9 de febrero de 2009; aceptado el 18 de mayo de 2010.

Diánoia, vol. LV, no. 65 (Noviembre 2010). 